\title{
Effect of a Long-Acting Somatostatin Analogue (BIM23014) on Proliferative Diabetic Retinopathy: A Pilot Study
}

\author{
M. McCOMBE, ${ }^{*}$ S. LIGHTMAN $\uparrow \dagger$ D. J. ECKLAND,${ }^{*}$ A. M. HAMILTON, \\ S. L. LIGHTMAN $\dagger$ \\ London
}

\begin{abstract}
Summary
A pilot study on the use of a continuous infusion of somatostatin, by subcutaneous pumps in the management of proliferative diabetic retinopathy is reported. Two patients out of eight with proliferative retinopathy demonstrated improvement. One patient demonstrated regression of disc new vessels and the other a reduced area of retinal capillary non-perfusion, both demonstrated by fluorescein angiography. Control patients showed worsening of fluorescein leakage over the observation period of four to six weeks whereas the other six patients given the somatostatin infusion did not demonstrate any deterioration.

The mechanism of action of somatostatin in this study is unknown but it is thought to have direct anti-angiogenic properties as well as inhibiting growth hormone secretion.
\end{abstract}

Numerous clinical reports have postulated growth hormone $(\mathrm{GH})$ as an aetiological agent in the pathogenesis of proliferative diabetic retinopathy. ${ }^{1-5}$ The aetiology of diabetic microvascular complications appears to be multifactorial and is poorly defined. It is widely agreed that poor metabolic control predisposes to these complications but the link between diabetic control and their development is not yet fully understood. Circulating $\mathrm{GH}$ has an effect on glucose metabolism causing insulin resistance with resultant glucose intolerance, hyperglycaemia and increased insulin requirement." Type I diabetic patients have been reported to produce increased levels of circulating $\mathrm{GH}^{8}$, especially during periods of poor metabolic control. ${ }^{7}$

In 1953 Poulsen reported an improvement in proliferative diabetic retinopathy in a patient who suffered a post-partum pituitary infarction. ${ }^{5}$ Following this observation, therapeutic pituitary ablation was introduced ${ }^{1,4}$ to produce regression of severe proliferative retinopathy. The degree of regression obtained was reported to be directly related to the degree of pituitary ablation and $\mathrm{GH}$ deficiency produced ${ }^{y .10}$ Further observations lending support to the postulated relationship between $\mathrm{GH}$ and diabetic retinopathy followed. Retinopathy was reported as absent or only mild, in pituitary dwarfs with diabetes. ${ }^{2}$ In poorly controlled diabetics raised $\mathrm{GH}$ levels are invariably found ${ }^{11}$ and an abnormal increase in GH secretion has also been noted with exercise ${ }^{12}$ and during sleep. This is the same group in which diabetic retinopathy is more likely to be found.

Selective pharmacological inhibition of $\mathrm{GH}$

From: *Moorfields Eye Hospital, City Road, London EC1V 2PD. †Westminster Hospital, Horseferry Road, London SW1.

Correspondence to: Professor S. Lightman, Moorfields Eye Hospital, City Road, London EC1V 2PD. 
secretion has therefore been suggested as a method by which regression of proliferative retinopathy may be obtained as well as resulting in an improvement in the metabolic control of Type I diabetes. ${ }^{13.14}$

Somatostatin is a hypothalamic polypeptide that inhibits the secretion of several peptide hormones including $\mathrm{GH}$, glucagon and insulin in man. ${ }^{15.16}$ It is widely distributed throughout the nervous system, gastrointestinal tract and pancreas and also delays the gastrointestinal absorption of carbohydrates. ${ }^{17}$ The administration of somatostatin to patients with Type 1 diabetes ${ }^{15}$ results in a marked reduction in their requirement for exogenous insulin. Natural somatostatin has been isolated and successfully used to inhibit GH secretion in $\operatorname{man}^{18}$ but its very short plasma half life of two to three minutes makes it unsuitable for long-term clinical use.

The somatostatin analogue Octreotide (SMS 201-995) has a longer half-life and its duration of action in man is six to eight hours. In an early study ${ }^{9}$ with this analogue, $\mathrm{GH}$ levels were reduced by $50 \%$ and insulin requirements decreased by $28 \%$ in Type I diabetics. GH was however, not completely suppressed. Hyer and others ${ }^{19}$ failed to suppress GH secretion completely with s/c injections over three days. Three days of continuous s/c pump infusion of Octreotide completely suppressed $\mathrm{GH}$ in normal subjects but only resulted in partial suppression of GH in Type I diabetics with retinopathy. There have been few other reports of the use of somatostatin analogues in Type I diabetics. There have been no reports of complete suppression of $\mathrm{GH}$ in diabetic retinopathy and the effect of incomplete $\mathrm{GH}$ suppression on diabetic retinopathy has been reported in only one series. ${ }^{19}$ Treatment did not prevent recurrent retinal haemorrhages and further laser treatment was necessary in all patients.

Pan-retinal laser photocoagulation is the current treatment of choice for proliferative retinopathy. The benefit of this treatment has been confirmed in a randomised clinical trial, the diabetic retinopathy study (DRS). ${ }^{20,21}$ Argon laser pan-retinal photocoagulation is however a destructive treatment and visually significant side-effects are reported. ${ }^{21,22,25-27}$ These include decreased visual acuity due to macular oedema, ${ }^{21.25 .26}$ peripheral visual field loss, ${ }^{21,25}$ temporary loss of foveal contrast sensitivity $^{27}$ and reduced night vision. ${ }^{26}$ There is also a group of patients with medial opacities, either vitreous haemorrhage, cataract or corneal opacities, in which laser can be difficult or in some cases, impossible to apply.

The aim of this pilot study was to assess a new long-acting somastatin analogue, BIM23014, (Somatuline-IPSEN BIOTECH, Paris, France) as an alternative treatment, rather than as an adjunct, to panretinal laser photocoagulation for the management of proliferative diabetic retinopathy. BIM23014 has a plasma half-life of 90 minutes following a single subcutaneous injection of $1000 \mu \mathrm{g}$ in healthy patients (Investigator's Manual, IPSEN). BIM23014 injected subcutaneously at a dose of $400 \mu \mathrm{g}$ followed by a continuous infustion of $1600 \mu \mathrm{g}$ has been reported to completely suppress $\mathrm{GH}$ plasma levels in healthy volunteers, ${ }^{23-24}$ while in acromegalic patients, a dose of $1500 \mu \mathrm{g} /$ day produces $\mathrm{GH}$ levels of less than $5 \mu \mathrm{g} / \mathrm{ml}$ in $98 \%$ of patients. (Investigator's Manual, IPSEN).

\section{Patients and Methods}

Seventeen adult patients with insulin dependent diabetes and proliferative retinopathy were recruited. Six of these patients were controls. As the study was a pilot project, the patients were not randomised. The age range of these patients was 25 to 58 years.

The inclusion criteria were:

(1) insulin dependent diabetes,

(2) proliferative retinopathy where the neovascular tissue was in isolated patches in the periphery in less than two quadrants in the periphery or occupied less than or equal to a quarter of the disc with no vitreous or pre-retinal haemorrhage (low risk-DRS definition)

(3) ability to modify own dose of insulin.

The exclusion criteria were:

(1) florid proliferative retinopathy, greater than two quadrants or involving more than a quarter of the disc,

(2) proliferative retinopathy with either vitreous or pre-retinal haemorrhage (high risk-DRS definition),

(3) non-insulin dependent diabetic patients,

(4) opaque media where clinical and angio- 
graphic assessment was rendered less reliable,

(5) laser treatment applied within three months prior to recruitment,

(6) females of child-bearing age and not contracepted.

The first two exclusion categories were judged as requiring urgent laser photocoagulation and not able to wait for an interval of four weeks for laser treatment if still required. Informed written consent was obtained from all patients recruited and the protocol for the study was approved by the Ethical Committees of Moorfields Eye Hospital and Westminster Hospital.

Initial assessment was performed at Moorfields Eye Hospital in the Diabetic Retinal Clinic. The assessment included colour retinal photography and fluorescein angiography. The patients were then seen at the Westminster Hospital by the diabetologist where a full medical work-up was carried out. The course of BM23014 treatment was then commenced. Eleven patients recruited were given $\mathrm{BM} 23014,1500 \mu \mathrm{g} /$ day, via a continuous subcutaneous infusion pump (Ferring BTZ). The patients were treated for a total of three months. Diabetic control was monitored by blood glucose measurements and haemaglobin A1 assays. Serum GH $(\times 4$ at each visit $)$, and Insulin-like growth (factor-1 (IGF-1) assays were performed as part of the study.

All patients were then reviewed at Moorfields Eye Hospital at four weeks following commencement of BIM23014, then at six and/or eight weeks and 12 weeks. Clinical assessment of the retina was repeated as were colour fundus photography and fluorescein angiography. The control group patients were seen at the same time intervals and had the same assessment as the patients given BIM23014. There was no significant difference in diabetic control between the subjects and the controls. Rather than a complete fundus photographic survey being undertaken to obtain serial frames of an area of proliferation, one or two areas of interest were identified in one eye and these same areas were photographed at each examination. ${ }^{1}$ Frames from each angiogram, taken at the same time in the fluorescein run, were then compared. In particular, the early frames were the most useful as the area to be studied was not swamped with fluorescein. At the first four week visit the eye was treated with argon laser panretinal photocoagulation (PRP) if there was no significant regression of retinal neovascularisation clinically or angiographically. Angiographic results/reports were based on a subjective assessment of the alteration in fluorescein leakage at a specified site.

\section{Results}

Eleven patients were commenced on the continuous infusion pump. In this group, $\mathrm{GH}$ was found to be completely suppressed in daytime assays. Plasma levels of BIM23014 were always above $3 \mathrm{ng} / \mathrm{ml}$. Levels of more than 1.5 $\mathrm{ng} / \mathrm{ml}$ have been shown to completely suppress $\mathrm{GH}$ in studies on acromegalic patients. Pretreatment levels of IGF-1 were below the normal range $(0.42+/-0.06$ units $/ \mathrm{ml})$ and further suppression was obtained with treatment $(0.27+/-0.04$ units $/ \mathrm{ml})$ after six weeks. This further suppression suggests significant reduction of $\mathrm{GH}$ secretion. Insulin requirements were reduced on average by $20-25 \%$, but despite this HbA-1 levels were unaffected $(10.7+/-0.8 \%$ before and $9.43+/-0.67 \%$ after treatment). Despite the diminished insulin requirements, no patient reported hypoglycaemic episodes. Some of the patients did complain of abdominal discomfort and increased bowel movements but this usually improved during the course of the study.

Eight patients continued with the subcutaneous infusion pumps for at least four weeks. Two patients then withdrew. One withdrew following repeated skin infections at the pump cannula site and another due to difficulty managing the pump.

Three patients withdrew prior to completing the first four weeks due to difficulty in managing the pump.

Visual acuity did not alter in either the control group or in the group receiving pump treatment over the period of the trial. Vitreous or preretinal haemorrhage did not occur over the first four weeks prior to laser treatment in either group.

Of the eight patients who received Somatuline via subcutaneous pumps, two showed clinical and angiographic improvement and were not treated with laser at four weeks. 
These two patients are described in detail below. The remaining six showed no evidence of improvement at the four week assessment and consequently received laser treatment at four to six weeks following the commencement of Somatuline. There was no deterioration either clinically or angiographically in these six patients prior to laser treatment. Over the same period in the control group three of the six patients showed evidence of worsening leakage of fluorescein but there was no alteration angiographically in areas of capillary closure. Ophthalmoscopically there was no change in the degree of neovascularisation in the control group patients.

The two cases, described in detail, show evidence of improvement with the Somatuline infusion.

Case A. M.S. was 48 yr old insulin dependent diabetic for 17 years. Four to six months prior to enrolment in the study she had received focal laser treatment to the right eye. The patient presented with new vessels at the optic disc in the right eye. Figure 1a is a fluorescein angiogram taken at this time. The neovascularisation was approximately a quarter of the disc diameter. Two weeks post-commencement of Somatuline there was no obvious change in intensity of fluorescein but the area of leakage was smaller. At six weeks

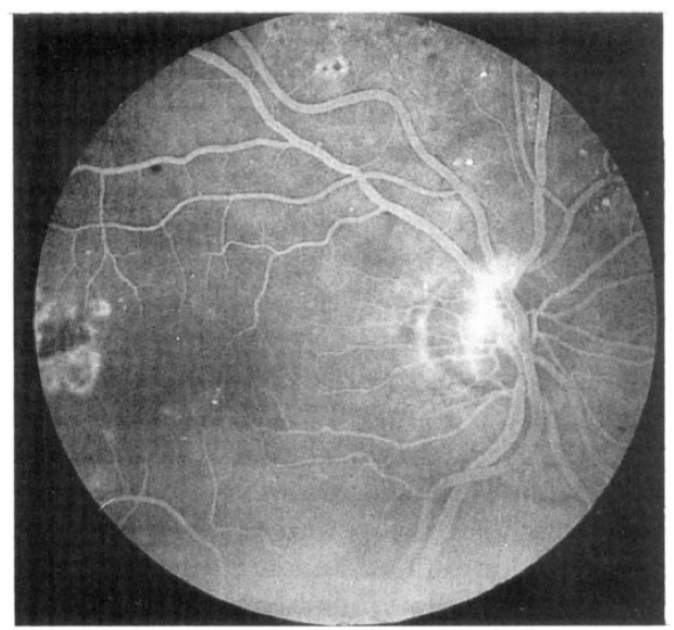

Fig. 1a. Patient M.S. Fluorescein angiogram at presentation. A patch of new vessels is present at the disc. Old laser scars are seen temporally, a result of a small amount of focal treatment for macular oedema a year before.
post-BM23014 commencement, the area and intensity of fluorescein leak at the disc was markedly less (Figure 1b). The six week angiograms are shown in Figure 1b. Clinically the new vessels were smaller and appeared less active.

Case B. B.L. was a 52 year old male who had been an insulin dependent diabetic for 21 years. He had bilateral PRP between February 1989 and April 1989. At the initial examination extensive PRP scars were present in both eyes with neovascularisation present at both discs. The right eye was examined by fluorescein angiography (Figure $2 \mathrm{a}$ ). There was no evidence of pre-retinal or vitreous haemorrhage in either eye and ophthalmoscopically the new vessels at the disc remained unchanged. Angiograms performed after four weeks of Somatuline (Fig. 2b) showed a mild reduction in the area of fluorescein leakage from the disc new vessels when compared to pre-treatment films. More noticeably there was less capillary non-perfusion on the posttreatment angiogram in areas temporal and superonasal to the disc.

\section{Discussion}

Eight of the 11 patients, commenced on the BM23014 infusion pumps, were evaluated at four weeks by ophthalmoscopic and angiographic examination. During this four to six week period no laser treatment was given. In

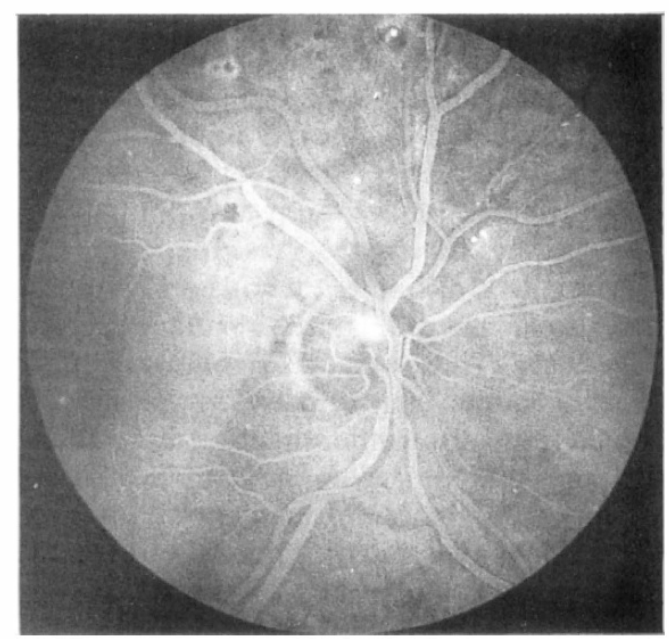

Fig. 1b. Patient M.S. Fluorescein angiogram at six weeks. The area of new vessels at the disc is much smaller with correspondingly less fluorescein leakage. 


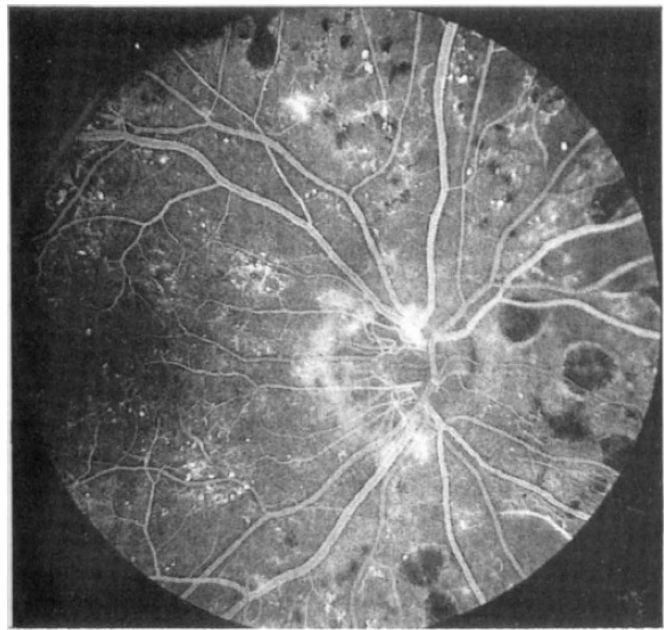

Fig. 2a. Patient B.L. Fluorescein angiogram at presentation. Fluorescein is seen leaking from disc new vessels. Also present are old laser scars, areas of capillary non-perfusion and temporal to the disc are patches of microaneurysms and capillary leakage.

two patients there was an improvement on angiographic assessment in the state of the proliferative retinopathy at this review. One patient showed a marked regression of new vessels and in the other the most obvious change was a reduction in the capillary leakage. The remaining six patients did not show any improvement but also did not deteriorate. These were all treated with PRP in the usual way. Although these patients continued with BM23014 (Somatuline) the angiograms taken following laser were of less value when attempting to assess the effect of Somatuline as it could be argued that any subsequent improvement was related to the laser treatment.

Improvement on Somatuline occurred in two out of eight patients which is a higher proportion than would have been expected to spontaneously or naturally regress without treatment.

With PRP established as an effective widely available treatment for proliferative retinopathy, somatostatin analogue therapy would have to display a greater success rate than is suggested in this pilot study. However PRP is a destructive treatment with side-effects and does have limitations as a form of treatment in some clinical situations. The numbers in this study are too small to draw a statistical con-

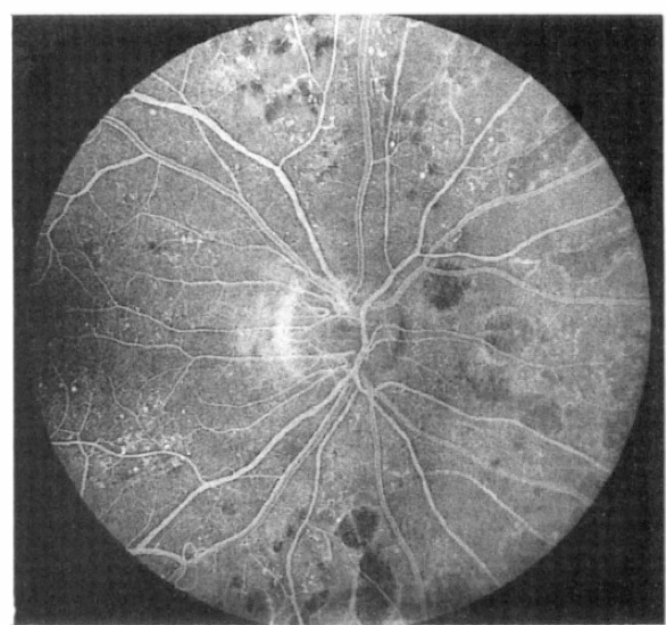

Fig. 2b. Patient B.L. At four weeks there is less capillary non-perfusion in areas temporal and superonasal to the disc. Mild reduction in leakage from the disc new' vessels.

clusion. Despite this, it is interesting to note that there was no deterioration in the retinopathy in the treated group whereas in the control group, three of the six patients showed increased fluorescein leakage at the four week review.

In this study, IGF-1 levels were suppressed by Somatuline. However, it has not been unequivocally shown that somatostatin analogues act on the pituitary to effect any improvement in retinopathy. Indeed somatostatin analogues may act at the retinal level to inhibit the activity of GH and IGF-1 locally rather than at the level of the pituitary. Recently, somatostatin analogues have been reported to inhibit angiogenesis in the chick chorioallantoic membrane. ${ }^{28}$ If the main inhibitory action does occur peripherally at the level of the retina, then an adequate therapeutic dose to improve retinopathy may not be related to that suppressing $\mathrm{GH}$, and may need to be higher than that used in this study.

Another potential area of study would be the role of Somatuline as an adjunct to PRP rather than as a replacement for it. Patients in which PRP can not be applied or applied only in a limited fashion, due to vitreous haemorrhage, media opacity or patient intolerance to laser, may be helped by a trial of pharmacological inhibition of growth hormone.

In summary, in a pilot study we were able to 
demonstrate improvement of proliferative retinopathy in two cases on the basis of fluorescein angiography following treatment with Somatuline for four weeks. Until further studies are undertaken on GH inhibition with somatostatin analogues, PRP still remains the treatment of choice for proliferative diabetic retinopathy.

Key words: diabetes, growth hormone, laser, proliferative diabetic retinopathy, somatostatin.

\section{References}

${ }^{1}$ Lundbaek K, Malmos R, Andersen HC, Rasmussen JH, Bruntse E, Madsen PH, Jensen VA: Hypophysectomy for diabetic angiopathy: a controlled clinical trial. In Goldberg MF, and Fine SL, eds: Symposium on the treatment of diabetic retinopathy, US Public Health Service Pub no 1890 Washington DC, 1969, US Government Printing Office.

${ }^{2}$ Merrimee TJ: A follow-up study of vascular disease in growth hormone deficient dwarfs with diabetes. N Engl J Med 1978, 298: 1217-22.

${ }^{3}$ Passa R, Rousselie F, Gauville C, Canivet J: Retinopathy and plasma growth hormone levels in idiopathic haemochromatosis with diabetes. Diabetes 1977, 26: 113-20.

${ }^{4}$ Kohner EM, Joplin GF, Blach RK, Cheng H, Fraser TR: Pituitary ablation in the treatment of diabetic retinopathy (a randomised trial). Trans Ophthalmol Soc UK 1972, 92: 79-90.

${ }^{5}$ Poulsen JE: Recovery from retinopathy in a case of diabetes with Simmond's disease. Diabetes 1953, 2: 7-12.

${ }^{6}$ Rizza RA, Mandarino LJ, Gerich JE: Effects of growth hormone on insulin action in man: mechanisms of insulin resistance, impaired suppression of glucose production, and impaired stimulation of glucose utilisation. Diabetes 1982, 31: 663-9.

${ }^{7}$ Gerich JE, Lorenzi M, Bier DM: Effects of physiological levels of glucagon and growth hormone on human carbohydrate and lipid metabolism: studies involving administration of exogenous hormone during suppression of endogenous hormone secretion with Somatostatin. J Clin Invest 1976, 57: 875-84.

${ }^{8}$ Hansen AP and Johansen K: Diurnal patterns of blood glucose, serum fatty acids, glucagon and growth hormone in normals and juvenile diabetics. Diabetologia 1970, 6: 27-33.

${ }^{9}$ Adams D, Rand R, Roth N, Dashe A, Gipstein R, Heuse G: Hypophysectomy in diabetic retinopathy. The relationship between degree of pituitary ablation and ocular response. Diabetes 1974 , 23: $698-707$.

${ }^{10}$ Wright AD, Kohner EM, Oakley NW, Hartog M, Joplin GF, Fraser TR: Serum GH and the response of diabetic retinopathy to pituitary ablation. $B r$ Med J 1969, 2: 246-8.

${ }^{11}$ Holly JMP, Amiel SA, Sandhu RR, Rees LH, Wass JAH: The role of growth hormone in diabetes mellitus. J Endocrinol 1988, 118: 353-64.
${ }^{12}$ Passa P, Gauville C, Canivet J: Influences of muscular exercise on plasma level of growth hormone in diabetics with and without retinopathy. Lancet 1974, 2: 72-4.

${ }^{13}$ Gerich JE: Rationale for inhibition of growth hormone secretion in the management of the diabetic patient. Scand J Gastroenterol (Suppl) 1986, 119: 154-7.

${ }^{1+}$ Plewe G, Noelken G, Krause U, Beyer J, del Pozo E: Suppression of growth hormone and somatomedin $\mathrm{C}$ by long-acting somatostatin analogue SMS 201-995 in type 1 diabetes mellitus. Hormone Res 1987, 27: 7-12.

${ }^{15}$ Gerich JE, Lorenzi M, Schneider V, Karam JH, Rivier J, Guillemin R, Forsham PH: Effects of somatostatin on plasma glucose and glucagon levels in human diabetes mellitus. New Engl J Med 1974, 291: 544-7.

${ }^{16}$ Gerich JE, Schultz TA, Lewis SB, Karam JH: Clinical evaluation of somatostatin as a potential adjunct to insulin in the management of diabetes mellitus. Diabetologia 1977, 13: 537-44.

${ }^{17}$ Wahren $\mathbf{J}$ and Felig P: Influence of somatostatin on carbohydrate disposal and absorption in diabetes mellitus. Lancet 1976, 2: 1213-16.

${ }^{18}$ Hall R, Besser GM, Schally AV: Action of growthhormone-release inhibitory hormone in healthy men and in acromegaly. Lancet 1973, 2: 581-4.

${ }^{14}$ Hyer SL, Sharp PS, Brooks RA, Burrin JM, Kohner EM: Continuous subcutaneous octreotide infusion markedly suppresses IGF-1 levels whilst only partially suppressing GH secretion in diabetics with retinopathy. Acta Endocrinol (Copenhagen) 1989, 120: 187-94.

21) Diabetic Retinopathy Study Research Group: Preliminary report on effects of photocoagulation therapy. Am J Ophthalmol 1976, 81: 383-96.

${ }^{21}$ Diabetic Retinopathy Study Research Group: Photocoagulation treatment of proliferative diabetic retinopathy: the second report of Diabetic Retinopathy Study findings. Ophthalmology 1978, 85: 82-106.

22 Diabetic Retinopathy Study Research Group: Photocoagulation treatment of proliferative diabetic retinopathy: clinical application of Diabetic Retinopathy Study (DRS) findings, DRS report no. 8. Invest Ophthalmol Vis Sci 1981, 88: $583-600$.

${ }^{23}$ Sassolas G, Khalfallah Y, Chayvaille JA: Effects of the Somatostatin analogue BIM 23014 on the secretion of growth hormone, thyrotropin and digestive peptides in normal men. J Clin Endocrinol Metab 1989, 68: 239-46.

${ }^{2+}$ Kuhn JM, Basin C, Molard M, Rouge de B, Wolf LM: Effects of the new somatostatin analog $(B / M$ 20314) on glucose homeostasis in normal men. The Endocrine Society, 72nd Annual Meeting. June 1990 p 51 (abstract).

${ }^{25}$ Diabetic Retinopathy Study Research Group: Indications for photocoagulation treatment of diabetic retinopathy: DRS Report No. 14. Int Ophthalmol Clin 1987 (Winter); 27(4): 239-53.

${ }^{26}$ McDonald HR and Schatz H: Visual loss following panretinal photocoagulation for proliferative diabetic retinopathy. Ophthalmology 1985, 92: 388-93.

${ }^{27}$ Higgins KE, Meyers SM, Jaffe MJ, Roy MS, Monas- 
terio FM: Temporary loss of foveal contrast sensitivity associated with panretinal photocoagulation. Arch Ophthalmol 1986, 104: 997-1003.

${ }^{28}$ Woltering EA, Barrie R, O’Doriso TM, Arce D,
Ure T, Cramer A, Holmes D, Robertson J, Fassler J: Somatostatin analogues inhibit angiogenesis in the chick allantoic membrane. Digestion 1990. 8th International symposium on Gastro-intestinal Hormones p 123. 